\title{
RETRACTED ARTICLE: Short hairpin RNA gene silencing of NLRP3 confers protection against sepsis-induced hyperbilirubinemia in a rat model
}

Yin Wu • Jianan Ren · Song Liu • Bo Zhou •

Chao Ding · Jun Chen · Gefei Wang •

Guosheng Gu $\cdot$ Jieshou Li

Published online: 31 December 2014

(C) Springer Science+Business Media New York 2015

The authors hereby retract this article as they have acknowledged publishing results derived from cell line experiments that they were unable to reproduce.

Y. Wu $\cdot$ J. Ren $(\bowtie) \cdot$ S. Liu $\cdot$ B. Zhou $\cdot$ C. Ding $\cdot$ J. Chen G. Wang $\cdot$ G. Gu $\cdot$ J. Li

Department of Surgery, Jinling Hospital, Medical School of Nanjing University, 305 East Zhongshan Road, Nanjing 210002, People's Republic of China

e-mail: JiananR@gmail.com 